\title{
Number of Premature Live Births
}

National Cancer Institute

\section{Source}

National Cancer Institute. Number of Premature Live Births. NCI Thesaurus. Code C139266.

A measurement of the total number of live birth events at which the gestational age of the neonate is less than 37 weeks and 0 days. 